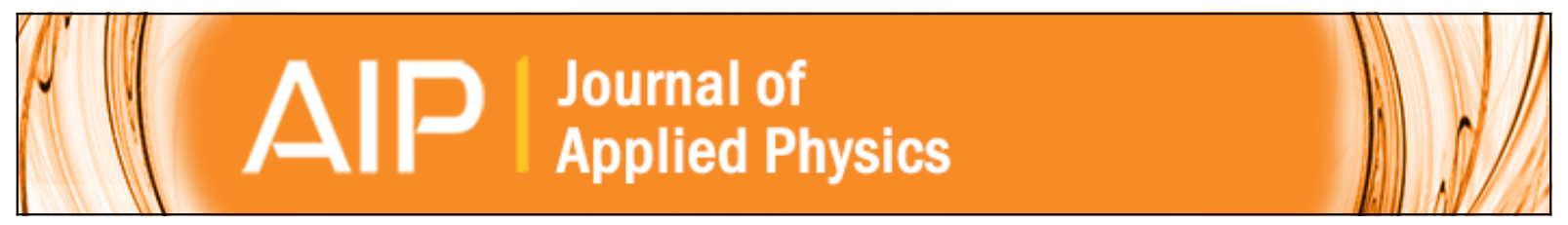

\title{
Structural and optical properties of InGaN-GaN nanowire heterostructures grown by molecular beam epitaxy
}

F. Limbach, T. Gotschke, T. Stoica, R. Calarco, E. Sutter, J. Ciston, R. Cusco, L. Artus, S. Kremling, S. Höfling,

L. Worschech, and D. Grützmacher

Citation: Journal of Applied Physics 109, 014309 (2011); doi: 10.1063/1.3530634

View online: http://dx.doi.org/10.1063/1.3530634

View Table of Contents: http://scitation.aip.org/content/aip/journal/jap/109/1?ver=pdfcov

Published by the AIP Publishing

\section{Articles you may be interested in}

Germanium doping of self-assembled GaN nanowires grown by plasma-assisted molecular beam epitaxy J. Appl. Phys. 114, 103505 (2013); 10.1063/1.4820264

Micro-Raman investigations of InN-GaN core-shell nanowires on $\mathrm{Si}$ (111) substrate AIP Advances 3, 062114 (2013); 10.1063/1.4811365

Molecular beam epitaxial growth and optical properties of red-emitting $(\lambda=650 \mathrm{~nm}) \operatorname{lnGaN} / \mathrm{GaN}$ disks-innanowires on silicon

Appl. Phys. Lett. 102, 071101 (2013); 10.1063/1.4793300

Molecular beam epitaxy growth and optical properties of AIN nanowires

Appl. Phys. Lett. 96, 061912 (2010); 10.1063/1.3315943

InN nanocolumns grown by plasma-assisted molecular beam epitaxy on A -plane GaN templates

Appl. Phys. Lett. 94, 221908 (2009); 10.1063/1.3151824

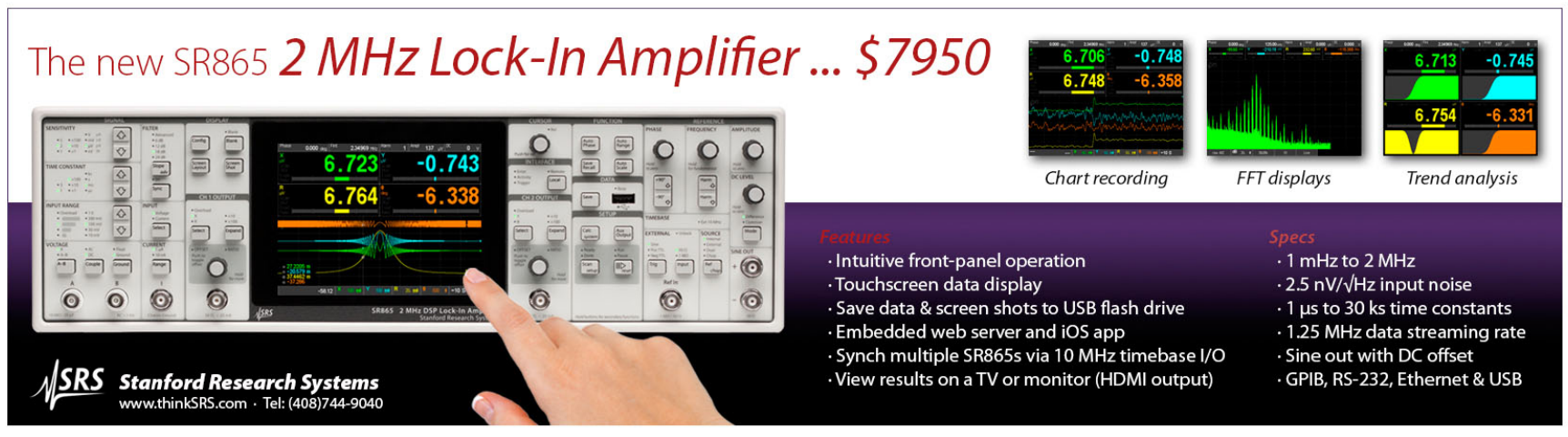




\title{
Structural and optical properties of InGaN-GaN nanowire heterostructures grown by molecular beam epitaxy
}

\author{
F. Limbach, ${ }^{1, a)}$ T. Gotschke, ${ }^{1}$ T. Stoica, ${ }^{1}$ R. Calarco, ${ }^{1}$ E. Sutter,${ }^{2}$ J. Ciston, ${ }^{2}$ R. Cusco, ${ }^{3}$ \\ L. Artus, ${ }^{3}$ S. Kremling, ${ }^{4}$ S. Höfling, ${ }^{4}$ L. Worschech, ${ }^{4}$ and D. Grützmacher ${ }^{1}$ \\ ${ }^{1}$ Institute of Bio- and Nanosystems (IBN-1), Research Centre Jülich GmbH and JARA-FIT Fundamentals \\ of Future Information Technology, 52425 Jülich, Germany \\ ${ }^{2}$ Center for Functional Nanomaterials, Brookhaven National Laboratory, Upton, New York 11973, USA \\ ${ }^{3}$ Institut Jaume Almera, Consell Superior d'Investigacions Científiques (CSIC), 08028 Barcelona, \\ Catalonia, Spain \\ ${ }^{4}$ Wilhelm Conrad Rontgen Research Centre Complex Matter Systems, University of Wurzburg, \\ D-97070 Wurzburg, Germany
}

(Received 23 September 2010; accepted 17 November 2010; published online 7 January 2011)

\begin{abstract}
$\mathrm{InGaN} / \mathrm{GaN}$ nanowire (NW) heterostructures grown by plasma assisted molecular beam epitaxy were studied in comparison to their $\mathrm{GaN}$ and $\mathrm{InGaN}$ counterparts. The InGaN/GaN heterostructure NWs are composed of a GaN NW, a thin InGaN shell, and a multifaceted InGaN cap wrapping the top part of the GaN NW. High-resolution transmission electron microscopy (HRTEM) images taken from different parts of a InGaN/GaN NW show a wurtzite structure of the $\mathrm{GaN}$ core and the epitaxial InGaN shell around it, while additional crystallographic domains are observed whithin the InGaN cap region. Large changes in the lattice parameter along the wire, from pure GaN to higher In concentration demonstrate the successful growth of a complex InGaN/GaN NW heterostructure. Photoluminescence (PL) spectra of these heterostructure NW ensembles show rather broad and intense emission peak at $2.1 \mathrm{eV}$. However, $\mu$-PL spectra measured on single NWs reveal a reduced broadening of the visible luminescence. The analysis of the longitudinal optical phonon Raman peak position and its shape reveal a variation in the In content between $20 \%$ and $30 \%$, in agreement with the values estimated by PL and HRTEM investigations. The reported studies are important for understanding of the growth and properties of NW heterostructures suitable for applications in optoelectronics and photovoltaics. () 2011 American Institute of Physics. [doi:10.1063/1.3530634]
\end{abstract}

\section{INTRODUCTION}

In the past two decades the Ga-Al-In-N material system, also called group III-nitrides, has revolutionized solid state light emitters ${ }^{1}$ and has impacted photovoltaics as well as high-power and temperature electronics. ${ }^{2,3}$ The InGaN ternary system offers band gap tunability covering the whole visible spectral range. ${ }^{4,5}$ Moreover, InGaN nanostructures and nanowires (NWs), in particular, may provide a pathway to integrating optoelectronic devices on $\mathrm{Si}^{6}{ }^{6}$ III-nitride NWs can be grown as single nanocrystals with high crystalline perfection on $\mathrm{Si}(111)$ and (100), as well as on amorphous $\mathrm{SiO}_{2}$ substrates. ${ }^{7-9}$ The small footprint of the NWs on the substrate greatly relaxes issues related to the structural mismatch between substrate and NW. Many publications have already reported on the growth of homoepitaxial III-nitride NWs by plasma assisted molecular beam epitaxy (PAMBE),${ }^{10-20}$ including InGaN NWs on Si substrates. ${ }^{21,22}$ However, to exploit the whole range of optoelectronic devices compatible with a NW design, the possibility to realize axial as well as radial hetero- and quantum structures is essential. So far these efforts have focused on introducing thin quantum disks into the NWs (Refs. 13 and 23-32) with the aim of fabricating NW light emitting diodes (LEDs). In this paper, we report on the growth of InGaN/GaN NW heterostructures with both axial and radial components prepared by

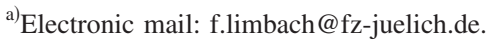

PAMBE and on their structural and optical properties. These heterostructures contain extended InGaN segments exceeding the few nanometers usually used in quantum well approaches. These detailed studies aid in the understanding of the growth mechanisms that govern the inclusion of extended InGaN sections in GaN NWs that may prove to be useful in NW LEDs. ${ }^{33}$

\section{EXPERIMENT}

The InGaN/GaN heterostructure NWs were grown by PAMBE on $\mathrm{Si}(111)$ substrates in nitrogen rich conditions without any catalyst. The silicon substrates were cleaned before epitaxy by a standard ex situ chemical cleaning procedure and by in situ annealing in ultrahigh vacuum at $925^{\circ} \mathrm{C}$ for $15 \mathrm{~min}$, in order to obtain an oxygen-free surface. The GaN NW base was grown at a substrate temperature of $T_{S}$ $=785^{\circ} \mathrm{C}$, a Ga beam equivalent pressure of $\mathrm{BEP}_{\mathrm{Ga}}=5.0$ $\times 10^{-8}$ mbar, an rf-power of $\mathrm{P}_{\mathrm{rf}}=500 \mathrm{~W}$, a nitrogen flow rate of 4 SCCM (SCCM denotes cubic centimeter per minute at STP) and a growth duration of $2 \mathrm{~h}$. Subsequently, the growth was interrupted and $\mathrm{T}_{\mathrm{S}}$ ramped down to $600{ }^{\circ} \mathrm{C}$ within $10 \mathrm{~min}$. Thereafter, the InGaN growth was started for $2 \mathrm{~h}$ with $\mathrm{BEP}_{\mathrm{Ga}}=3.0 \times 10^{-8} \mathrm{mbar}$ and $\mathrm{BEP}_{\mathrm{In}}=3.4$ $\times 10^{-8}$ mbar with the same nitrogen plasma conditions. The two reference samples shown in Figs. 1(b) and 1(a) were grown under the same growth conditions used for the GaN NW base and InGaN cap, respectively. 

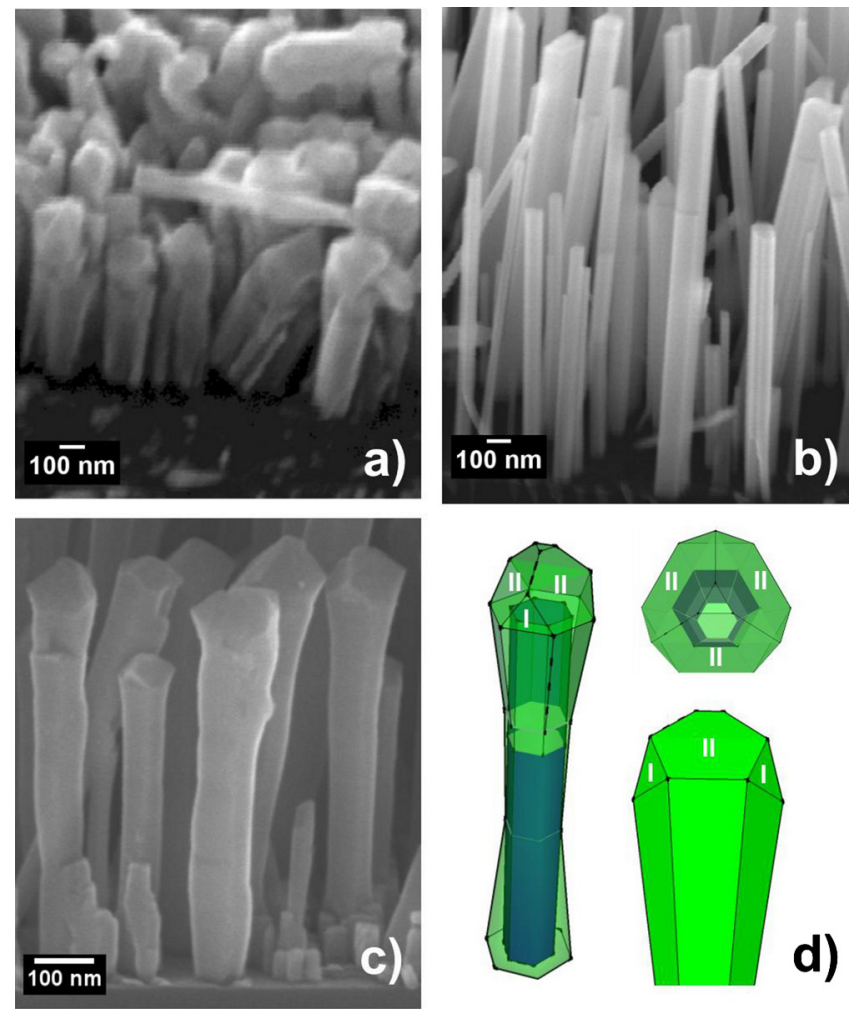

d)

FIG. 1. (Color online) (a) SEM of InGaN grown directly on $\mathrm{Si}$ at $600{ }^{\circ} \mathrm{C}$. (b) SEM of pure GaN NWs grown at $785^{\circ} \mathrm{C}$. (c) SEM of a InGaN/GaN heterostructure, the materials were grown for $2 \mathrm{~h}$ each. (d) Sketch of the NW morphology according to analysis.

Investigation of the morphology of the NWs was carried out in a Zeiss Leo1550 scanning electron microscope (SEM), a JEOL-2100F field-emission transmission electron microscope (TEM) operated at $200 \mathrm{kV}$, and an FEI Titan fieldemission TEM with third order image aberration corrector operated at $300 \mathrm{kV}$. The composition of the NWs was investigated in scanning TEM (STEM) mode with an energy dispersive x-ray (EDS) detector. The In/Ga concentration was quantified from the EDS spectra using the integrated peak intensities (In-L and Ga-L) and library element standards provided by the OXFORD ENERGY TEM software package. Tomographic tilt series over a 130 degree range were collected in STEM mode with a detector annular radius of 30-65 mrad. The NWs were mechanically severed from the substrate and dispersed on a carbon film supported by a standard $\mathrm{Cu}$ grid. The cross-sectional TEM samples were prepared by focused ion beam and tripod polishing. Photoluminescence (PL) measurements were performed using the 325 $\mathrm{nm}$ line of a $\mathrm{Cd}-\mathrm{He}$ Laser as an excitation source, on as grown samples mounted in a liquid $\mathrm{He}$ cooled cryostat. Spectral analysis of the PL signal was carried out with a SPEX 1702 monochromator and a charge coupled device (CCD) detector camera. For the $\mu$-PL measurements the asgrown wires were dispersed mechanically onto a thermally oxidized $\mathrm{Si}(001)$ substrate $\left(100 \mathrm{~nm} \mathrm{SiO}_{2}\right)$. The substrate was additionally structured by e-beam lithography with Au markers in order to locate individual wires. Raman scattering measurements were performed at room temperature in backscattering geometry using a Jobin-Yvon T64000 spectrom- eter equipped with a CCD detector cooled with liquid nitrogen. The excitation wavelength used was the $488 \mathrm{~nm}$ line of an $\mathrm{Ar}^{+}$laser.

\section{RESULTS AND DISCUSSION}

\section{A. NW morphology and structural properties}

The morphology of the samples was investigated by SEM. The SEM side view images in Figs. 1(a) and 1(b) show pure InGaN and GaN NWs, respectively. The InGaN and GaN NWs were grown as control samples with which the InGaN/GaN heterostructures, shown in (c), could be compared. It can be seen [Fig. 1(a)] that the InGaN NWs have a small aspect ratio of about 4 and no symmetric hexagonal shape is evident from SEM top view images. The InGaN NWs slightly enlarge toward the top and show a general coalescence tendency. In contrast to the InGaN NWs, both GaN NWs and the heterostructure NWs have much higher aspect ratios and exhibit faceting with hexagonal shape consistent with the top basal plane and growth along the c-axis. However, it can be seen that the heterostructure NWs increase in diameter toward the top whereas the GaN NWs have smooth and very parallel sidewalls, i.e., uniform diameter over the entire length of the GaN NW. Moreover, the GaN NWs have a flat top surface while the InGaN/GaN NWs exhibit a complex apex with a threefold symmetry. Three of the six sidewalls are terminated in triangular shaped sidewalls labeled I in the morphology scheme in Fig. 1(d) (the blue hexagonal core represents the initially grown $\mathrm{GaN}$ while the green shell is formed during the subsequent InGaN growth). The hexagonally shaped facets labeled II are inclined toward the center of the wire and form a tripod pyramid. The complex morphology is similar to the one observed by Kishino et al. $^{34}$ but differs from the findings by Hong et al. ${ }^{35}$ where the NW tops were seen to be almost flat.

To investigate further the overall morphology and the complex apex of the heterostructure NWs, high resolution TEM investigations were performed. Figure 2(a) shows a cross-sectional TEM image of an array of InGaN/GaN NWs. In contrast to the pure GaN NWs, the InGaN/GaN NWs exibit a gradual increase in diameter toward the tops [as seen by SEM, Fig. 1(c) as well]. A caplike structure with a facetted apex is visible at the top part of the NWs. A somewhat smaller increase in diameter is seen close to the base of the heterostructure NWs as well as some additional small wires adjacent to the substrate between the longer ones. The change in diameter of the longer NWs can be attributed to the onset of InGaN growth on the GaN NWs. The small NWs most likely also nucleated in the InGaN phase of the growth $^{13}$ as they show the same tendency toward coalescence as is observed for pure InGaN NWs [SEM image in Fig. 1(a)]. In Fig. 2(b) a higher maginification TEM image from the middle section of a heterostructure NW and the corresponding diffraction pattern (DP) are shown. The DP can be indexed to the GaN wurtzite structure growing along the c-axis. The simulated DP along the [2 $\overline{1} \overline{1} 0]$ zone axis is shown for comparison in color (slightly shifted to the right from the experimental spots). It can be seen that the diffraction spots stemming from this part of the NW are double, 

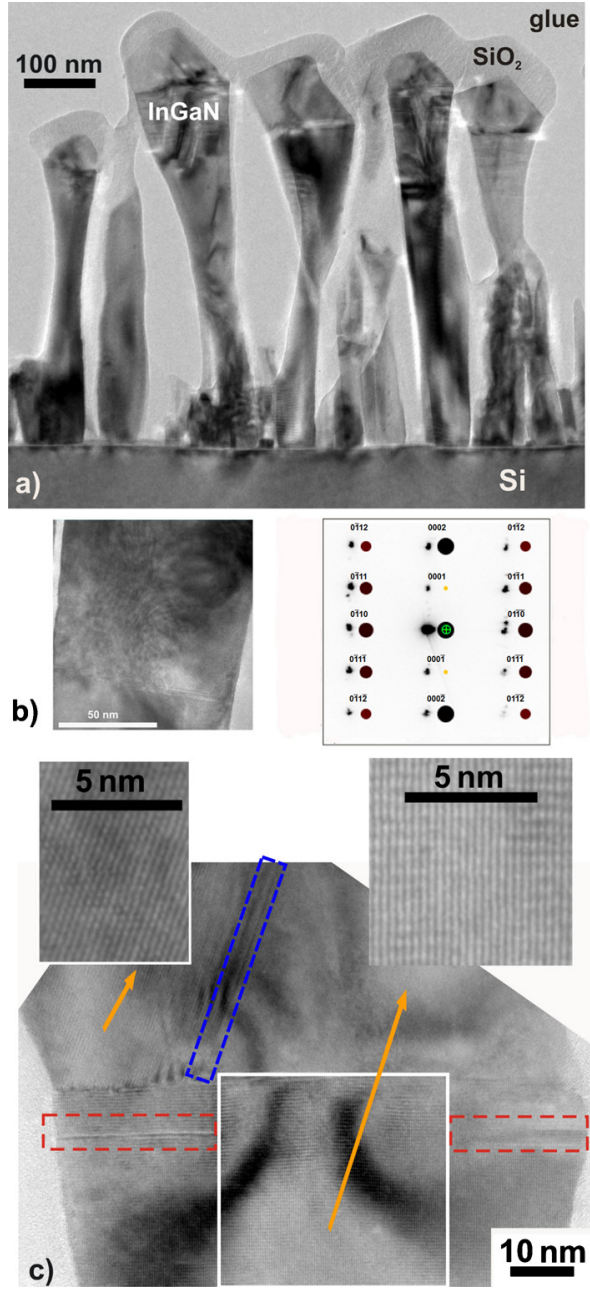

FIG. 2. (Color online) (a) TEM cross section of the InGaN/GaN NW ensamble. (b) TEM of the top region of an InGaN/GaN heterostructure NW and the corresponding DP. (c) HRTEM of the top region of an $\mathrm{InGaN} / \mathrm{GaN}$ wire the white box indicates the previously grown GaN, stacking faults can be observed in the areas in the dashed boxes. The insets show TEM pictures of two different regions in the NW.

i.e., the electron beam probes regions of material with two close but different lattice spacings. This would be the case if the electron beam penetrates through a thin but well-defined shell of InGaN (larger lattice constant than $\mathrm{GaN}$ ) grown epitaxially on the GaN core.

The cross-sectional high resolution TEM image in Fig. 2(c) shows a high resolution image of the cap at the heterostructure NWs. Surprisingly, the facetted apex of the wire has a different crystallographic orientation than the lower parts. This is highlighted in the insets of Fig. 2(c) showing lattice images of the trunk and cap of the NW. The DP from the cap shows that there still is a signal of the GaN core present in the cap but the InGaN alloy has now a growth direction inclined by $66^{\circ}$ relative to the original direction. The inclination of the InGaN cap fluctuates from wire to wire, with inclinations of the terminating facets leading to shape variation. The interface between the two distinct parts of the NW having different crystallographic orientation is clearly indicated by the termination of inclined stacking faults [marked by a blue rectangle in Fig. 2(c)]. In addition, the cross section depicts two defects one on either side of the image, which extend approximately $25 \mathrm{~nm}$ from the side surface into the wire [marked by the red boxes in Fig. 2(c)]. The position at which these defects terminate appears to coincide with the surface of the previously grown GaN NW. We conclude that discrete InGaN alloy covers the top part of the GaN NW and forms a complex cap, schematically shown in Fig. 1(d). The formation of the complex cap in all NWs is observed to follow for a region of epitaxial growth of $\sim 15 \mathrm{~nm}$. For illustration the white line in Fig. 2(c) indicates the interface between the GaN trunk and the InGaN cap. Thus, there is an axial interface at the top and a radial interface at the sides of the GaN trunk. The InGaN grows on the sidewalls at the upper part of the GaN trunk forming a coreshell region and on the top facet of the $\mathrm{GaN}$ core forming a faceted cap.

A careful structural investigation of the NW in Fig. 3(a) was performed, in which high-resolution TEM (HRTEM) images from $10 \times 10 \mathrm{~nm}^{2}$ regions along different parts of the wire were analyzed using fast Fourier transform (FFT) to evaluate the spacings of the crystallographic planes of the $\mathrm{InGaN}$ and the $\mathrm{GaN}$ core and their relative orientation. The areas of FFT analysis were taken from regions with no visible stacking faults or other extended defects, even though in the parts of the NW close to the top such defects are present, as the presence of such defects and strain fluctuations in neighboring areas can influence local lattice spacings. These effects, as well as the local residual strain are neglected in our computation of the In concentration profile. The InGaN growing on the sidewalls, forming the shell around the trunk has the same crystal orientation as the GaN. Within this coreshell region of the wire, InGaN is deposited nonuniformly, resulting in NWs with constantly increasing diameter toward the top. In the lower part of the NW the smallest diameter of about $25 \mathrm{~nm}$ is found, which corresponds approximately to the GaN NW core diameter. The plane spacing fluctuates in a wide range along the wire. As can be seen in Fig. 3(a), there are regions with lattice distances close to $5.18 \AA$, the value of pure $\mathrm{GaN}$ (position 1 and 2 of the GaN core), as well as regions with larger plane spacing up to $5.43 \AA$. However, the most frequent value determined in the shell region is about $5.26 \AA$ as for the points 2 and 3 in Fig. 3(a), which corresponds to $\sim 16 \%$ In content in an unstrained alloy. If some compressive strain remains, the In concentration is underestimated by this procedure. The strain of a thin InGaN layer with $20 \%$ In concentration deposited on the lateral sides of the GaN NW can be estimated at about $2 \%$. The local In concentration has a tendency to increase and strongly fluctuate toward the top of the core-shell NW region, reaching values as high as $\sim 50 \%$ In at point 5 .

In the InGaN cap which grows on the top of the GaN wire, domains with different crystallographic orientations can be identified. In the particular TEM image of Fig. 3(a), two domains are visible. These are indicated by white straight lines in Fig. 3(a). One domain (L-left part) has the $\mathrm{c}$-axis in the same plane as the rest of the wire, but rotated approximately $70^{\circ}$ relative to the $\mathrm{GaN} \mathrm{NW}$ growth direction and the estimated lattice distance of $5.26 \AA$ corresponds to an alloy containing $22 \% \mathrm{In}$. The other region ( $\mathrm{R}$-right part) of the cap might be a wurtzite structure with the c-axis almost 

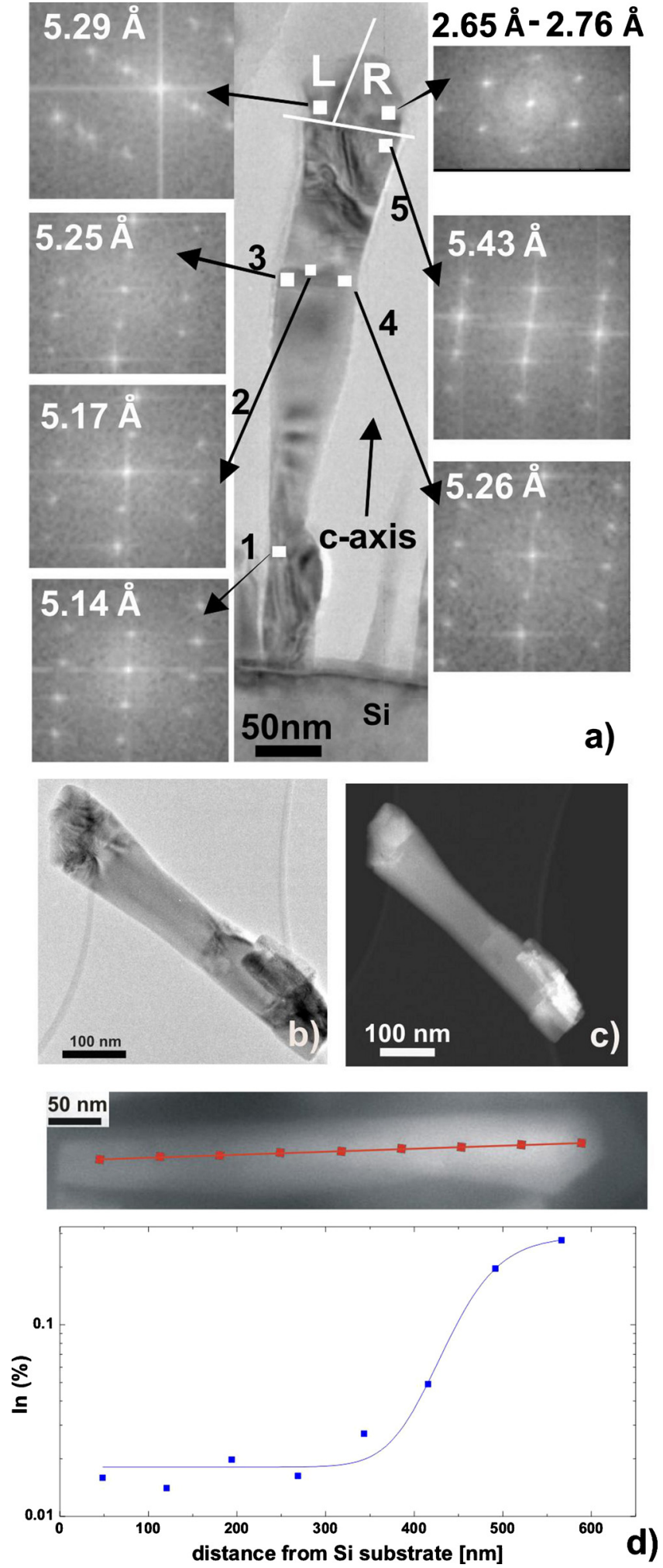

FIG. 3. (Color online) (a) Local FFT results from indicated positions along the wire and the corresponding c-lattice parameters. (b) TEM image of a single wire on a copper grid. (c) Still image of a Z-contrast movie of the same wire shown in (b). (d) STEM image of InGaN/GaN heterostructure indicating where local EDS spectra have been taken (red boxes). The graph gives the percentage of In with respect to $\mathrm{Ga}$ in the beam path as a function of the distance from the Si substrate.

perpendicular to the c-axis of the rest of the wire, or may be of cubic type. As previously discussed, the formation of dif- ferent crystallographic domains determines the faceted shape of the InGaN cap, which frequently exibitins a threefold symetry.

Figures 3(b) and 3(c) show the TEM image and the annular dark field image of a single heterostructure NW, respectively. The interface between the trunk and the cap is clearly visible [upper left corner in Fig. 3(c)] due to Z-contrast indicating higher In content in the cap compared to the remaining NW (see below for details). At the base of the NW (lower left corner in Fig. 3(c) brighter contrast is visible, which originates from three small wires attached to the longer one. This brighter contrast results from two factors- $\mathrm{i}$, due to this agglomeration the e-beam penetrates through more material, which gives rise to higher number of scattered electrons at the annular detector angle and ii. The shorter NWs at the base nucleated later than the fully grown NWs during the InGaN growth as discussed above and probably contain more In than the amount at the base of the fully grown NW. Importantly, in Fig. 3(c) brightness contrast change is observed along the growth axis of the NW, which indicates that the interface between the initially grown GaN NW and the subsequent InGaN growth is sharp (see also Ref. 41).

In order to evaluate the In concentration EDS measurements were carried out in STEM mode with a spot size of about $0.2 \mathrm{~nm}$. Figure 3(d) shows a representative bright-field STEM image of an InGaN/GaN NW heterostructure along which EDS line profiles were taken. The points at which the EDS measurements were performed are marked by colored dots. The In content as a function of the distance from the $\mathrm{Si}$ substrate is shown in Fig. 3(d). The In concentration is evaluated as a mean value which includes the contribution due to the GaN core. Overall In was detected over the entire length of the NW even though the distribution is not uniform. While the middle section of the wire contains very little In in the cap the mean concentration of In increases rapidly and reaches about 27 at. \%.

\section{B. Optical properties}

The PL spectra of the three samples shown in Fig. 1 are presented in Fig. 4(a). The InGaN NWs show a very broad PL emission [full width at half maximum (FWHM $=0.76 \mathrm{eV})]$ centered at $2.1 \mathrm{eV}$. However, no emission was observed in the near band edge region of $\mathrm{GaN}$ around 3.47 $\mathrm{eV}$. This broad peak could be assigned to $\mathrm{InGaN}$ alloy band edge emission but also to defects present in $\mathrm{GaN}$. If spontaneous phase seperation takes place, GaN NWs or GaN inclusions in InGaN could have also been produced. Due to the low growth temperature a high density of structural defects can be expected, which would cause the PL emission at 2.1 $\mathrm{eV}$. In order to rule this hypothesis out, a GaN sample grown at the same growth parameters without In supply was investigated, and no luminescence at $2.1 \mathrm{eV}$ could be detected. The PL spectrum of the GaN NWs shown in Fig. 1(b) has a strong band edge emission that contains free exciton $\mathrm{X}_{\mathrm{A}}$ and donor bound exciton $\mathrm{D}^{0} \mathrm{X}_{\mathrm{A}}$ contributions. ${ }^{9}$ In addition emission between 3.1 and $3.26 \mathrm{eV}$ is observed, which is typically assigned to a donor acceptor pair (DAP) transition. ${ }^{6}$ 

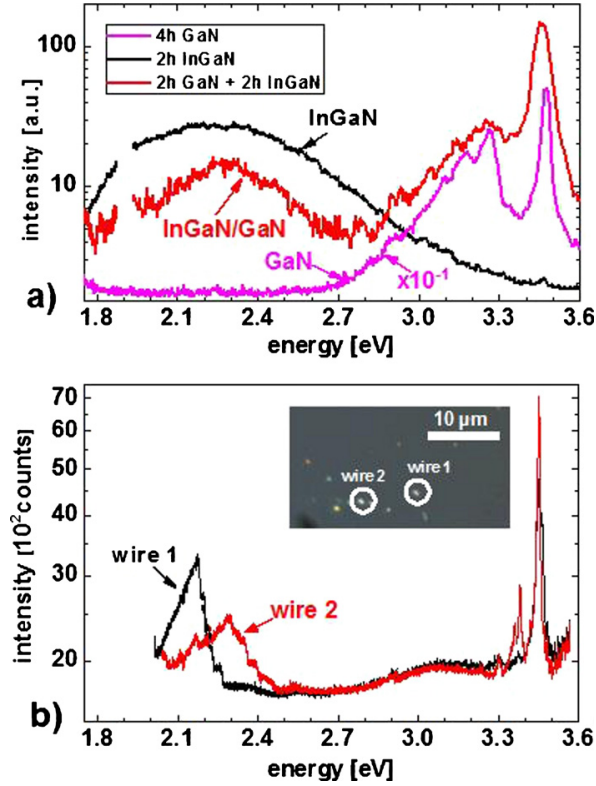

FIG. 4. (Color online) (a) PL spectra for the samples shown in Fig. $1(\mathrm{a})-1(\mathrm{c})$ at $160 \mathrm{~K}$, deleted points are due to second harmonic of the excitation laser. (b) $\mu$-PL measurements of two single NWs with the positions indicated in the inset (optical microscope).

The PL spectrum of InGaN/GaN NW heterostructure also shown in Fig. 4(a) has the characteristics of both spectra $\mathrm{GaN}$ and InGaN NWs described previously. In the spectral range of the emission from the GaN core, the GaN near band edge peak as well as the DAP region can be observed. The broad emission from the $\mathrm{InGaN}$ is centered at about $2.2 \mathrm{eV}$ with a FWHM of $0.8 \mathrm{eV}$. The peak position suggests an average incorporation of approximately $30 \% \mathrm{In}^{6}{ }^{6}$ in agreement with EDS measurements. The broadness of the emission could be explained by compositional variations in the InGaN alloy along the NW and between NWs. This is confirmed by $\mu$-PL measurements on single InGaN/GaN NW heterostructures presented in Fig. 4(b). In total 21 wires have been investigated, all showing luminescence near the GaN band edge region. Most NWs also exhibit luminescence below $2.6 \mathrm{eV}$. The center of this emission varies from wire to wire and has a FWHM as low as $0.25 \mathrm{eV}$. This broadening is assigned to band gap variation due the different In content in the core-shell and cap segments of individual wires. A variation in the In content of $8 \%$ corresponds to the FWHM of $0.25 \mathrm{eV}$. This variation in the In content indicated by $\mu$-PL is in agreement with that of the TEM analysis discussed previously.

In order to additionally confirm the In concentration derived from PL measurements and to rule out green defect luminescence of the GaN (Refs. 6, 36, and 37) Raman measurements were conducted exciting the sample using the 488 $\mathrm{nm}$ line of an $\mathrm{Ar}^{+}$laser. As a reference, spectra from GaN and InGaN NW samples were also obtained and compared with the InGaN/GaN NW heterostructure sample. The Raman spectra of the NW samples are displayed in Fig. 5. For comparison, the Raman spectrum of a metal organic chemical vapor deposition (MOCVD)-grown InGaN thin film with $[\mathrm{In}]=24 \%$ is also shown. After subtraction of the luminescence background, a clear Raman peak emerges in the spec-

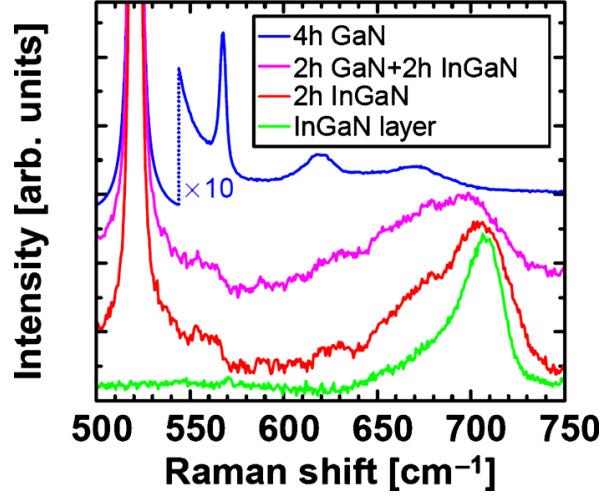

FIG. 5. (Color online) Room temperature Raman spectrum of the InGaN/ GaN NW heterostructure sample (color online magenta) compared to the spectra of the $\mathrm{GaN}$ and $\mathrm{InGaN}$ reference NW samples. The bottom spectrum corresponds to an MOCVD-grown InGaN layer with In concentration of $24 \%$.

tra of both, the InGaN/GaN and the InGaN NW samples at about $700 \mathrm{~cm}^{-1}$. This corresponds to longitudinal optical (LO) modes of the InGaN alloy, which are resonantly enhanced via the Fröhlich interaction under the near resonant conditions of the experiment. ${ }^{38}$ No feature associated with the $\mathrm{E}_{2}$ mode could be observed above the luminescence background. Although the experiments were carried out in backscattering configuration from the sample surface, given the morphology of the NWs (see Fig. 1), which exhibits a large proportion of lateral and beveled facets, the observed LO peak is probably due to quasi-LO modes with a strong component of the $\mathrm{E}_{1}(\mathrm{LO})$ mode (see for instance Ref. 39). However, the $\mathrm{A}_{1}(\mathrm{LO})$ and $\mathrm{E}_{1}(\mathrm{LO})$ modes are very close in frequency, ${ }^{40}$ and, therefore, a single LO peak is observed due to broadening of the Raman lines by alloying fluctuations. The frequency of the resulting quasi-LO mode gives a good indication of the mean value of the InGaN alloy composition. By comparison with the Raman spectrum of the InGaN layer, an average In composition in the InGaN NWs of $\sim 25 \%$ can be estimated according to the composition dependence of the $\mathrm{A}_{1}(\mathrm{LO})$ frequency. ${ }^{38}$ Raman measurements on the GaN NWs were performed away from resonance conditions and only a weak, sharp $\mathrm{E}_{2}$ peak was detected at $\sim 567 \mathrm{~cm}^{-1}$. The additional bands that can be observed in the Raman spectrum of that sample correspond to the first and second-order peaks of the $\mathrm{Si}$ substrate. In the $\mathrm{InGaN} /$ GaN NW heterostructure sample no GaN Raman signal could be detected as it was buried in the luminescence background.

The LO peak observed in the $\mathrm{InGaN}$ and $\mathrm{InGaN} / \mathrm{GaN}$ NW samples displays an asymmetrical broadening to the low frequency side. This probably reflects In composition fluctuations across the NWs (see above) and also within the ensemble of NWs probed by the Raman measurement. ${ }^{38}$ The LO peak of the InGaN/GaN NW heterostructure is clearly shifted to lower frequencies relative to that of the InGaN NW sample. Such a frequency shift could be related to a higher In content of the InGaN alloy in the NW heterostructure.

\section{CONCLUSIONS}

In conclusion InGaN/GaN NW heterostructures show a complex morphology with a hexagonal wire base and a tri- 
pod pyramidal top. The In content was evaluated combining TEM structural and compositional investigation and optical measurements. TEM investigations of the lattice spacing by FFT as well as DPs indicate variations in In content along the wire. Compared to EDS (In content: 27\%) both optical measurements provide a similar In content (Raman: 25\%/PL: $30 \%$ ). Single wire PL measurements confirm compositional variations from wire to wire and along the wire. This finding is also supported by Raman measurements of the NW ensemble.

\section{ACKNOWLEDGMENTS}

The authors wish to thank K. H. Deussen (IBN-1) and K. Kisslinger (Brookhaven National Laboratory) for technical support. This work was financially supported by the German Ministry of Education and Research project "QPENS." It was performed in part under the auspices of the U.S. Department of Energy, under Contract No. DE-AC02-98CH1-886. R.C. and L.A. acknowledge financial support from the Spanish Ministry of Science and Innovation under Contract No. MAT2007-63617. See Ref. 41 for a set of annular dark field images of single InGaN/GaN NW heterostructure are displayed in the electronic supplementary material as a movie extracted from a STEM tomography tilt series.

${ }^{1}$ E. F. Schubert and J. K. Kim, Science 308, 1274 (2005).

${ }^{2}$ J. Wu, W. Walukiewicz, K. M. Yu, W. Shan, J. W. Ager, E. E. Haller, H. Lu, W. J. Schaff, W. K. Metzger, and S. Kurtz, J. Appl. Phys. 94, 6477 (2003).

${ }^{3}$ P. Kung and M. Razeghi, Opto-Electron. Rev. 8, 201 (2000).

${ }^{4}$ S. Nakamura, S. Pearton, and G. Fasol, The Blue Laser Diode: The Complete Story (Springer-Verlag, Heidelberg, 2000).

${ }^{5}$ T. Kuykendall, P. Ulrich, S. Aloni, and P. Yang, Nature Mater. 6, 951 (2007).

${ }^{6} \mathrm{H}$. Morkoç, Handbook of Nitride Semiconductors and Devices (WileyVHC Verlag, Weinheim, 2008), Vol. 1-3.

${ }^{7}$ E. Calleja, M. A. Sánchez-Garciá, F. J. Sánchez, F. Calle, F. B. Naranjo, E. Muñoz, U. Jahn, and K. Ploog, Phys. Rev. B 62, 16826 (2000).

${ }^{8}$ R. Meijers, T. Richter, R. Calarco, T. Stoica, H. P. Bochem, M. Marso, and H. Lüth, J. Cryst. Growth 289, 381 (2006).

${ }^{9}$ N. Thillosen, K. Sebald, H. Hardtdegen, R. Meijers, R. Calarco, S. Montanari, N. Kaluza, J. Gutowski, and H. Luth, Nano Lett. 6, 704 (2006).

${ }^{10}$ L. Robins, K. A. Bertness, J. M. Barker, N. A. Sanford, and J. B. Schlager, J. Appl. Phys. 101, 113505 (2007).

${ }^{11}$ L. Cerutti, J. Ristic, S. Fernandez-Garrido, E. Calleja, A. Trampert, K. H. Ploog, S. Lazic, and J. M. Calleja, Appl. Phys. Lett. 88, 213114 (2006).

${ }^{12}$ J. Renard, R. Songmuang, C. Bougerol, B. Daudin, and B. Gayral, Nano Lett. 8, 2092 (2008).

${ }^{13}$ R. Calarco, R. J. Meijers, R. K. Debnath, T. Stoica, E. Sutter, and H. Lüth, Nano Lett. 7, 2248 (2007).
${ }^{14}$ T. Stoica, E. Sutter, R. Meijers, R. K. Debnath, R. Calarco, and H. Lüth, Small 4, 751 (2008).

${ }^{15}$ K. Goodman, K. Wang, X. Luo, J. Simon, T. Kosel, and D. Jena, MRS Symposia Proceedings No. 1080 (Materials Research Society, Pittsburgh, 2008), p. 1080-O08-04

${ }^{16}$ R. K. Debnath, R. Meijers, T. Richter, T. Stoica, R. Calarco, and H. Lüth, Appl. Phys. Lett. 90, 123117 (2007).

${ }^{17}$ R. Calarco and M. Marso, Appl. Phys. A: Mater. Sci. Process. 87, 499 (2007).

${ }^{18}$ T. Stoica, R. J. Meijers, R. Calarco, T. Richter, E. Sutter, and H. Lüth, Nano Lett. 6, 1541 (2006).

${ }^{19}$ T. Stoica, R. J. Meijers, R. Calarco, T. Richter, and H. Lüth, J. Cryst. Growth 290, 241 (2006).

${ }^{20}$ L. Geelhaar, C. Chèze, W. M. Weber, R. Averbeck, H. Riechert, T. Kehagias, P. Komninou, G. P. Dimitrakopulos, and T. Karakostas, Appl. Phys. Lett. 91, 093113 (2007).

${ }^{21}$ A. P. Vajpeyi, A. O. Ajagunna, K. Tsagaraki, M. Androulidaki, and A. Georgakilas, Nanotechnology 20, 325605 (2009).

${ }^{22}$ K. M. Wu, Y. Pan, and C. Liu, Appl. Surf. Sci. 255, 6705 (2009).

${ }^{23}$ K. Kishino, A. Kikuchi, H. Sekiguchi, and S. Ishizawa, Proc. SPIE 6473, 64730T (2007).

${ }^{24}$ J. Ristić, E. Calleja, A. Trampert, S. Fernandez-Garrido, C. Rivera, U. Jahn, and K. H. Ploog, Phys. Rev. Lett. 94, 146102 (2005).

${ }^{25}$ J. Renard, R. Songmuang, G. Tourbot, C. Bougerol, B. Daudin, and B. Gayral, Phys. Rev. B 80, 121305 (2009).

${ }^{26}$ Y. Kawakami, S. Suzuki, A. Kaneta, M. Funato, A. Kikuchi, and K. Kishino, Appl. Phys. Lett. 89, 163124 (2006).

${ }^{27}$ R. Armitage and K. Tsubaki, Nanotechnology 21, 195202 (2010).

${ }^{28}$ A. L. Bavencove, G. Tourbot, E. Pougeoise, J. Garcia, P. Gilet, F. Levy, B. Andre, G. Feuillet, B. Gayral, B. Daudin, and L. S. Dang, Phys. Status Solidi A 207, 1425-1427 (2010).

${ }^{29}$ H.-W. Lin, Y.-J. Lu, H.-Y. Chen, H.-M. Lee and S. Gwo, Appl. Phys. Lett. 97, 073101 (2010).

${ }^{30}$ Y.-L. Chang, J. L. Wang, F. Li, and Z. Mi, Appl. Phys. Lett. 96, 013106 (2010).

${ }^{31}$ Y. S. Park, M. J. Holmes, T. W. Kang, and R. A. Taylor, Nanotechnology 21, 115401 (2010).

${ }^{32}$ H. Sekiguchi, K. Kishino, and A. Kikuchi, Phys. Status Solidi C 7, 2374 (2010).

${ }^{33}$ W. Guo, M. Zhang, A. Banerjee, and P. Bhattacharya, Nano Lett. 10, 3355 (2010).

${ }^{34}$ K. Kishino, S. Hiroto, and K. Akihiko, J. Cryst. Growth 311, 2063 (2009).

${ }^{35}$ C. C. Hong, H. Ahn, C. Y. Wu, and S. Gwo, Opt. Express 17, 17227 (2009).

${ }^{36}$ L. Polenta, A. Cavallini, M. Rossi, R. Calarco, M. Marso, T. Stoica, R. Meijers, T. Richter, and H. Lüth, ACS Nano 2, 287 (2008).

${ }^{37}$ A. Cavallini, L. Polenta, M. Rossi, T. Richter, M. Marso, R. Meijers, R. Calarco, and H. Lüth, Nano Lett. 6, 1548 (2006).

${ }^{38}$ S. Hernández, R. Cuscó, D. Pastor, L. Artús, K. P. O’Donnell, R. W. Martin, I. M. Watson, Y. Nanishi, and E. Calleja, J. Appl. Phys. 98, 013511 (2005).

${ }^{39}$ H. Harima, J. Phys.: Condens. Matter 14, R967 (2002).

${ }^{40}$ E. Alarcón-Lladó, R. Cuscó, L. Artús, J. Jiménez, B. Wang, and M. Callahan, J. Phys.: Condens. Matter 20, 445211 (2008).

${ }^{41}$ See supplementary material at 10.http://dx.doi.org/10.1063/1.3530634 for the interface between the initially grown GaN NW and the subsequent InGaN growth. 